Revisión

\title{
Trazodona: ¿Nuevo enfoque selectivo para el tratamiento de los sofocos generados por la deprivación androgénica en el carcinoma prostático?
}

\author{
Santiago Vilar González*, Francesc Montañá Puig**, Sebastiá Sabater Martí*, \\ M $^{\mathrm{a}}$ Victoria Villas Sánchez*, $\mathrm{M}^{\mathrm{a}}$ del Mar Sevillano Capellán*, Manuel Aguayo Martos* \\ *Complejo Hospitalario Universitario de Albacete (CHUA) Albacete. ${ }^{* *}$ Angelini-Farmaceútica. España
}

\section{Resumen}

El protagonismo creciente del carcinoma prostático en las sociedades desarrolladas hace que tenga que ser muy tenido en cuenta el balance beneficio-perjuicio de los tratamientos prestados.

Dado el enfoque terapéutico cada vez más complejo del carcinoma prostático, hoy en día se requiere de una suma de conocimientos extensos.

La deprivación androgénica juega un papel principal en esta patología. El tratamiento de la toxicidad derivada en forma de sofocos, síndrome metabólico, osteoporosis, trastornos cognitivos, etc., adquiere cada vez mayor interés.

El tratamiento farmacológico de los sofocos pasa por un manejo hormonal no exento de riesgo oncológico a la par que de una toxicidad nada despreciable. Dentro del tratamiento no hormonal de esta patología juegan un papel destacado los antidepresivos.

La trazodona, un antidepresivo SARI (inhibidores de la recaptación de serotonina/antagonista de la 2A), con un perfil de actuación más selectivo sobre los receptores implicados en los sofocos; podría ser de gran interés. Trazodona muestra una gran afinidad por los receptores 5- $\mathrm{HT}_{2 \mathrm{~A}} \mathrm{y}$ una moderada afinidad por los receptores 5- $\mathrm{HT}_{1 \mathrm{~A}}$.

Como es conocido los niveles de serotonina (5-hidroxitriptamina o 5-HT) en mujeres postmenopáusicas están disminuidos, normalizándose con las terapias sustitutivas. Todo ello sugiere que la deprivación abrupta de hormonas sexuales da lugar a una reducción en la circulación de serotonina, con el consiguiente aumento de sus receptores 5- $\mathrm{HT}_{2 \mathrm{~A}}$ hipotalámicos. Estos receptores estarían implicados en la patogénesis de los sofocos siendo su bloqueo una de las principales medidas terapéuticas.

$\mathrm{El}$ uso de trazodona elevando las concentraciones de serotonina y bloqueando a los receptores 5- $\mathrm{HT}_{2 \mathrm{~A}}$ y 5 - $\mathrm{HT}_{1 \mathrm{~A}}$ podría plantearse como un nuevo enfoque más de acorde con la fisiopatología de los sofocos. Estudios comparativos bien dirigidos son necesarios para dar respuesta en cuanto a su efectividad. Otras cuestiones pendientes serían las dosis y el tiempo de tratamiento más eficaz para el control de los sofocos.

Palabras clave: Carcinoma prostático. Sofocos. Deprivación hormonal. Tratamiento. Antidepresivos. Trazodona. Receptores 5-HT2A .

\section{Trazodone: A new selective approach to the treatment of hot flashes induced by androgen deprivation in prostate carcinoma?}

\section{Abstract}

The growing relevance of prostate carcinoma in the developed world requires serious attention to focus on the risk-benefit relationships of the treatments used.

Given the increasingly complex therapeutic approach to prostate carcinoma, an extensive range of knowledge is required.

Androgen deprivation plays a central role in this disease. The management of androgen deprivation-derived toxicity in the form of hot flashes, metabolic syndrome, osteoporosis, cognitive disorders, etc., is of growing interest.

The drug treatment of hot flashes involves hormone management that is not without oncological risk and moreover generates considerable toxicity. Antidepressants in turn play an important role in the non-hormone treatment of this disorder.

Trazodone, a serotonin reuptake inhibitor $/ 5-\mathrm{HT}_{2 \mathrm{~A}}$ receptor antagonist affording more selective action upon the receptors implicated in hot flashes, could be of great interest. Trazodone shows great affinity for the $5-\mathrm{HT}_{2 \mathrm{~A}}$ receptors and moderate affinity for the $5-\mathrm{HT}_{1 \mathrm{~A}}$ receptors.

Serotonin (5-hydroxytryptamine, or 5-HT) levels are known to be lowered in postmenopausal women, and normalize when replacement therapy is provided. This suggests that abrupt sexual hormone deprivation gives rise to a reduction in blood serotonin with a subsequent increase in its hypothalamic $5-\mathrm{HT}_{2 \mathrm{~A}}$ receptors. These receptors would be implicated in the physiopathology of hot flashes; as a result, the blocking of such receptors is one of the principal therapeutic measures.

The use of trazodone, increasing the serotonin concentrations and blocking the 5- $\mathrm{HT}_{2 \mathrm{~A}}$ and $5-\mathrm{HT}^{1 \mathrm{~A}}$ receptors, could be viewed as a novel management approach more in line with the physiopathology of hot flashes. Well designed comparative studies are needed to establish the efficacy of such treatment. Other issues pending clarification would be the most effective dose and duration of treatment for controlling hot flashes.

Keywords: Prostate carcinoma. Hot flashes. Hormone deprivation. Treatment, Antidepressants. Trazodone. 5-HT2A receptors. 
T a mayor incidencia y prevalencia del carcinoma prostático en las sociedades desarrolladas, con una supervivencia previsible cada vez mayor; hace que deba ser tenido en cuenta, entre otras cuestiones, la yatrogenia generada.

La deprivación hormonal con análogos de la LH$\mathrm{RH}$ juega un papel principal en el tratamiento tanto de los pacientes afectos de carcinomas prostáticos localizados de alto riesgo como de los pacientes localmente avanzados y metastásicos. El papel que pueda jugar en los pacientes de riesgo intermedio ${ }^{1-}$ 3 pasa primero por consensuar por la comunidad científica la definición de los grupos de riesgo ${ }^{4}$, quedando también por aclarar el tiempo de mantenimiento. Su papel en adyuvancia a la braquiterapia también es otro punto de interés para estudios futu$\operatorname{ros}^{5,6}$.

Por todo ello, ante el número creciente de pacientes afectos de sofocos, se ha de incidir en su tratamiento.

La terapia hormonal sustitutiva no esta exenta

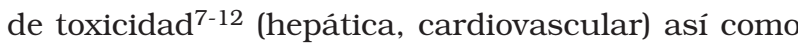
de un riesgo oncológico ${ }^{13,14}$, todo lo cual debe ser tenido en consideración.

Cualquier nueva terapia para la prevención y el tratamiento de la toxicidad inducida por la deprivación hormonal, basada en una terapia sustitutiva, debe asegurar su total seguridad en cuanto a la evolución del proceso neoplásico hormonodependiente.

Por todo ello y basándonos en la fisiopatología desencadenante de los sofocos, el enfoque no hormonal debe jugar un papel destacado, siendo hoy por hoy de primera elección.

La trazodona, un antidepresivo SARI, con un perfil de actuación muy selectivo sobre los receptores implicados en los sofocos; podría desempeñar un importante papel en su tratamiento.

\section{DESARROLLO}

A continuación revisaremos brevemente el mecanismo fisiopatológico implicado en el desarrollo de los sofocos.

Como se ha documentado la noradrenalina (NA) se presenta como el neurotransmisor implicado en el control del centro termorregulador, acortando su intervalo de respuesta y rebajando su umbral de tolerancia por lo que se genera una vasodilatación cutánea con sudoración profusa. Por ello en general los fármacos con función noradrenérgica posibilitarían la aparición de la sintomatología.
Por otra parte, mientras la NA genera este acortamiento del intervalo, la serotonina y la dopamina tienen un efecto contrario. Los niveles de serotonina (5-hidroxitriptamina o 5-HT) en mujeres postmenopáusicas están disminuidos, normalizándose con las terapias sustitutivas. Todo ello sugiere que la deprivación abrupta de hormonas sexuales da lugar a una reducción en la circulación de serotonina, con el consiguiente aumento de sus receptores $5-\mathrm{HT}_{2 \mathrm{~A}}$ hipotalámicos ${ }^{15,16}$. Estos receptores estarían implicados en la patogénesis de los sofocos ${ }^{15}$ por ello el uso de antidepresivos serotoninérgicos estaría justificado y de hecho se han ensayado varios de estos fármacos con resultados esperanzadores ${ }^{17-19}$.

Nuevos antidepresivos, en especial la venlafaxina y la paroxetina parecen ser los más eficaces en su control $^{17-21}$, con respuestas en torno al 50-65\%, algo menores que con las terapias hormonales pero con un perfil de seguridad mayor en los supervivientes con cáncer hormonosensible ${ }^{15}$.

Por otro lado la trazodona es una fenilpiperazina derivada de la triazolopiridina. Trazodona es un potente y selectivo antagonista postsináptico del receptor $5-\mathrm{HT}_{2 \mathrm{~A}}$ y un inhibidor moderado de la recaptación de serotonina ${ }^{22,23}$. Como se ha comentado es miembro del grupo de antidepresivos denominado SARI (inhibidores de la recaptación de serotonina/antagonista de la 2A). Muestra una gran afinidad por los receptores $5-\mathrm{HT}_{2 \mathrm{~A}} \mathrm{y}$ una moderada afinidad por los receptores $5-\mathrm{HT}_{1 \mathrm{~A}}{ }^{24}$ (Fig. 1).

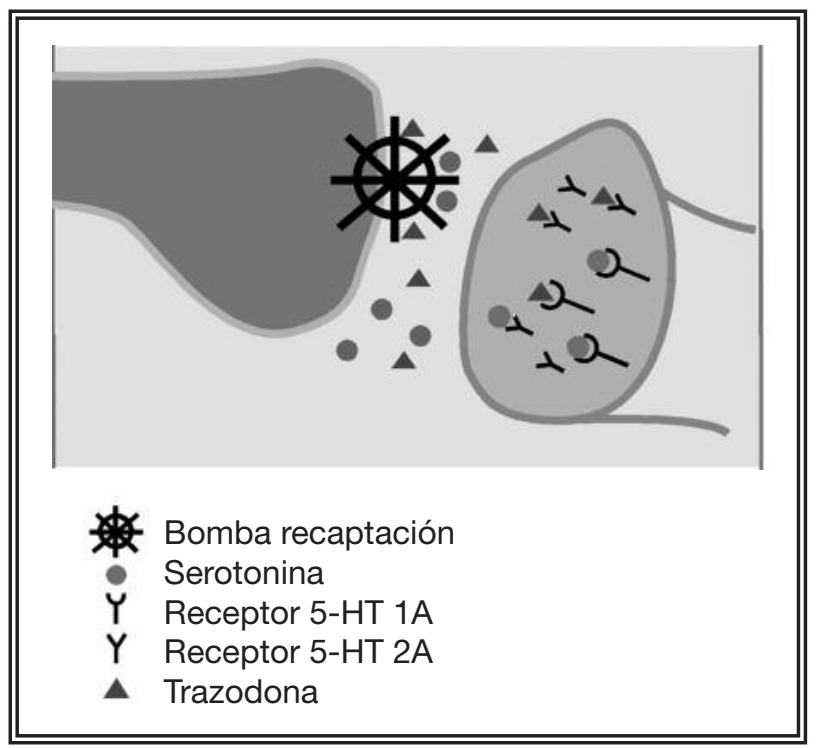

FIGURA 1. Mecanismo de actuación de trazodona a nivel sináptico. 
El perfil farmacológico de la trazodona se presenta interesante a la hora de valorar su uso en el tratamiento de los sofocos en pacientes con carcinoma prostático en tratamiento con deprivación hormonal.

La potencia de bloqueo de los receptores de serotonina $5-\mathrm{HT}_{2 \mathrm{~A}}$ varía considerablemente entre los antidepresivos de primera generación (tricíclicos). La importancia de dicho efecto sobre la acción terapéutica de dichos antidepresivos en general no está clara. Sin embargo, las fenilpiperazinas son más selectivas que los antidepresivos tricíclicos a la hora de bloquear a los receptores $5 \mathrm{HT}_{2 \mathrm{~A}}{ }^{24}$.

Si analizamos un ISRS (inhibidor selectivo de la recaptación de serotonina) clásico como la fluoxetina, ésta posee poca afinidad por los receptores 5$\mathrm{HT}_{1 \mathrm{~A}}$ y $5-\mathrm{HT}_{2 \mathrm{~A}}$, pero inhibe la recaptación de serotonina con mayor potencia que la trazodona. De hecho, la concentración necesaria del fármaco para producir una inhibición del 50\% $\left(\mathrm{CI}_{50}\right)$ de la recaptación es de sólo $6 \mathrm{nmol} / 1$ para fluoxetina, frente a $115 \mathrm{nmol} / 1$ para trazodona ${ }^{25,26}$ (Tabla 1).

La actividad de los ISRS produce una estimulación de todos los receptores de serotonina debido al aumento de los niveles libres de 5-HT. El no proporcionar un bloqueo específico de receptores $\mathrm{y}$, aunque esto ha demostrado ser útil en el tratamiento de la depresión, puede ser responsable de la estimulación de los receptores $5-\mathrm{HT}_{2 \mathrm{~A}}$ en algunas zonas del cerebro (p. ej. postencéfalo y la médula espinal), generalmente relacionados con la aparición de reacciones adversas al fármaco, tales como agitación, ansiedad, y disfunción sexual ${ }^{27}$. Recordemos que estos son los principales receptores implicados en los sofocos, y que sí son inhibidos por la trazodona.
Al mismo tiempo la trazodona no causa un bloqueo clínico de los receptores de acetilcolina y por lo tanto los efectos anticolinérgicos potenciales (visión borrosa, alteraciones cardiacas, sequedad bucal, trastornos intestinales, retención urinaria, aumento presión ocular) son irrelevantes. Tampoco presenta efecto clínico sobre la inhibición de la NA por lo que minimiza los posibles efectos secundarios tipo: apatía, falta de motivación, pérdida del placer y falta de concentración.

Por otro lado, trazodona presenta una afinidad interesante por los receptores $\alpha$. La alta y moderada actividad establecida de trazodona por los receptores adrenérgicos $\alpha_{1}$ y $\alpha_{2}$ respectivamente, podrían contribuir a sus efectos beneficiosos descritos en la disfunción eréctil ${ }^{28}$. Por el contrario pudiera plantear cierto riesgo ocasional de hipotensión ortostática.

También bloquea los receptores de histamina $\left(\mathrm{H}_{1}\right)$. Probablemente debido a este efecto y a su potente antagonismo $5-\mathrm{HT}_{2 \mathrm{~A}}{ }^{27,29}$ trazodona posee una actividad sedante que ha demostrado ser muy útil en el tratamiento de los pacientes ancianos con agitación e insomnio.

Por todo ello la trazodona no solamente puede controlar los sofocos sino que dado su perfil farmacológico nos podría favorecer mucho el control de la ansiedad, del sueño y de la disfunción eréctil con la consiguiente mejoría en la calidad de vida.

No están disponibles publicaciones de trazodona en pacientes con supresión hormonal y por lo que respecta a su uso frente a los sintomas de climaterio, únicamente se dispone de un estudio piloto con 25 pacientes, sin un diseño especifico, para valorar su eficacia en el control de los sofocos. Se utilizó a dosis de $75 \mathrm{mg} /$ día durante 3 meses y no se objetivo efecto sobre la puntuación de los sofocos pero sí

Tabla 1. Afinidades $\left(K_{\mathrm{i}}\right)$ de trazodona y otros antidepresivos por distintos receptores

\begin{tabular}{|c|c|c|c|c|c|}
\hline $\begin{array}{l}\text { Receptor } \\
\text { Radioligando } \\
\text { TejidoFuente }\end{array}$ & 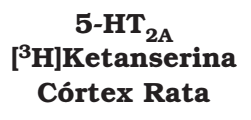 & $\begin{array}{c}5-\mathrm{HT}_{1 \mathrm{~A}} \\
{\left[{ }^{3} \mathrm{H}\right] 8-\mathrm{OH}-\mathrm{DPAT}} \\
\text { Hipocampo rata }\end{array}$ & 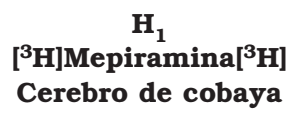 & $\begin{array}{c}\alpha 1 \\
\text { prazosina } \\
\text { Córtex Humano }\end{array}$ & $\begin{array}{c}\alpha 2 \\
{\left[{ }^{3} H\right] R a u w o l s c i n a} \\
\text { Córtex Humano }\end{array}$ \\
\hline Amitriptilina & $5,3 \pm 0,2$ & $129 \pm 2$ & $0,17 \pm 0,01$ & $4,4 \pm 0,2$ & $141 \pm 2$ \\
\hline Fluoxetina & $141 \pm 9$ & $8313 \pm 62$ & $933 \pm 23$ & $1,313 \pm 17$ & $3.090 \pm 121$ \\
\hline Nefazodona & $7,1 \pm 0,4$ & $52 \pm 1$ & $30 \pm 1$ & $5,5 \pm 0,3$ & $84 \pm 4$ \\
\hline Paroxetina & $6,320 \pm 608$ & $21,168 \pm 2401$ & $13,746 \pm 404$ & $995 \pm 35$ & $3.915 \pm 114$ \\
\hline Sertralina & $2,207 \pm 60$ & $3,663 \pm 82$ & $5,042 \pm 165$ & $36 \pm 2$ & $477 \pm 17$ \\
\hline Trazodona & $20 \pm 1$ & $42 \pm 1$ & $29 \pm 1$ & $12 \pm 0,2$ & $106 \pm 2$ \\
\hline Venlafaxina & $>100,000$ & $>100,000$ & $12.909 \pm 1,075$ & $39,921 \pm 810$ & $>100,000$ \\
\hline
\end{tabular}

Constantes de inhibición $\left(K_{\mathrm{i}} \mathrm{nmol} / \mathrm{l}-\right.$ media geométrica $\left.\pm \mathrm{DE}\right)$ (modificado de Owens ${ }^{26}$ ). 
en la reducción de la ansiedad, insomnio e irritabi$\operatorname{lidad}^{30}$. Aunque este estudio piloto no parece corroborar la posible eficacia de dicha molécula sobre los sofocos, cabe tener presente, que se llevó a cabo con una metodología mejorable en la actualidad.

Otro dato en contra de su eficacia en este estudio es la dosis utilizada, recordar su menor potencia en relación a los ISRS. Este punto podría soslayarse con un aumento de la dosis.

Dosis habituales de entre 200 a 400 mg sin riesgo alguno ${ }^{31}$, son utilizadas en la práctica clínica ambulatoria, reservándose dosis de hasta $600 \mathrm{mg}$ para el tratamiento de las depresiones con ingreso hospitalario ${ }^{32}$.

Dado el interesante perfil farmacológico y clínico de este fármaco sería deseable testar mediante metodología contrastada su capacidad de mejoría de los enfermos oncológicos prostáticos sometidos a deprivación hormonal, no solamente en el ámbito de los sofocos sino también en la mejoría de su calidad de vida, utilizando diversas escalas validadas que objetiven dicho efecto ${ }^{22,23}$.

Futuros estudios comparativos bien dirigidos son necesarios para obtener respuestas, no sólo en cuanto a su efectividad, sino también en cuanto a la dosis y el tiempo de tratamiento necesarios.

\section{REFERENCIAS}

1. D`Amico AV, Chen MH, Renshaw AA, Loffredo M, Kantoff PW. Androgen suppression and radiation vs radiation alone for prostate cancer: a randomized trial. JAMA. 2008;299(3):289-295.

2. Crook J, Ludgate C, Malone S, Lim J, Perry G, Eapen L, et al. Report of a multicenter Canadian phase III trial of 3 months vs 8 months neoadjuvant androgen before standard dose radiotherapy for clinically localized prostate cancer. Int J Radiation Oncol Biol Phys. 2004;60(1):15-23.

3. Denham JW, Steigler A, Lamb DS, Joseph D, Mameghan H, Turner S, et al. Short-term androgen deprivation and radiotherapy for locally advanced prostate cancer: results from the Trans-Tasman Radiation Oncology Group 96.01 randomised controlled trial. Lancet Oncol, 2005;6(11):841-850.

4. Ganswindt U, Paulsen F, Anastasiadis AG, Stenzl A, Bamberg M, Belka C. 70 Gy or more: which dose for which prostate cancer?. J Cancer Res Clin Oncol. 2005; 131(7):407-419.

5. Galalae RM, Martinez A, Mate T, Mitchell C, Edmundson G, Nuernberg N, et al. Long-term outcome by risk factors using conformal high-dose-rate brachytherapy (HDR-BT) boost with or without neoadjuvant androgen suppression for localized prostate cancer. Int $\mathrm{J}$ Radiat Oncol Biol Phys. 2004;58(4): 1048-1055

6. Jani AB, Shoushtari A, Feinstein JM. Role of androgen ablation with LowDose-Rate brachytherapy in the treatment of prostate cancer. Clin Drug Invest. 2006;26(12):723-731.

7. Loprinzi CL, Michalak JC, Quella SK, O’Fallon JR, Hatfield AK, Nelimark RA, et al. Megestrol acetate for the prevention of hot flushes. N Engl J Med. 1994;331(6):347-352.

8. Quella SK, Loprinzi CL, Sloan JA, Vaught NL, DeKrey WL, Fischer T, et al. Long-term use of megestrol acetate by cancer survivors for the treatment of hot flashes. Cancer. 1998;82(2): 1784-1788.

9. Loprinzi CL, Johnson PA, Jensen M. Megestrol acetate for anorexia and cachexia. Oncology, 1992;49:Suppl 2:46-49.

10. Miller JI, Ahmamn FR. Treatment of castration-induced menopausal symp toms with low dose diethylstilbestrol in men with advanced prostate cancer Urology. 1992;40(6):499-502.
11. Atala A, Amin M, Harty JI. Diethylstilbestrol in treatment of postorchiectomy vasomotor symptoms and its relation-ship with serum follicle-stimulating hormone, luteinizing hormone, and testosterone. Urology. 1992;39(2)108-110.

12. Moon TD. Cyproterone acetate for treatment of hot flushes after orchidectomy. Letter. J Urol. 1985;134(1):155-156.

13. Dawson N, McLeod DG. Dramatic prostate specific antigen decrease in response to discontinuation of megestrol acetate in advanced prostate of cancer: expansion of the antiandrogen withdrawal syndrome. J Urol. 1995;153 (6):1946-1947.

14. Wehbe TW, Stein BS, Akerley WL. Prostate-specific antigen response to withdrawal of megestrol acetate in a patient with hormone-refractory prostate cancer. Mayo Clin Proc. 1997;72(10):932-934.

15. Albertazzi P. Nonadrenergic and serotonergic modulation to treat vasomotor symptoms. J Br Menopause Soc. 2006;12(1):7-11.

16. Curcio JJ, Kim LS, Wollner D, Pockaj BA. The potential role of 5-Hydroxytryptophan for hot flash reduction: A hypothesis. Altern Med Rec. 2005 10(3):216-221.

17. Loprinzi CL, Barton DL, Carpenter LA, Sloan JA, Novotny PJ, Gettman MT, et al. Pilot evaluation of paroxetine for treating hot flashes in men. Mayo Clin Proc. 2004;79(10):1247-1251.

18. Quella SK, Loprinzi CL, Sloan J, Novotny P, Perez EA, Burch PA, et al. Pilot evaluation of venlafaxine for the treatment of hot flashes in men undergoing androgen ablation therapy for prostate cancer. Journal of Urology. 1999;162 (1):98-102.

19. Loprinzi CL, Kugler JW, Sloan JA, Mailliard JA, LaVasseur BI, Barton DL, et al. Venlafaxine in management of hot flashes in survivors of breast cancer: a randomized controlled trial. Lancet. 2000;356(9247):2059-2063.

20. (Epub ahead of print). Venlafaxine versus clonidine for the treatment of hot flashes in breast cancer patients: a double-blind, randomized cross-over study. Breast cancer res Treat, 2008 Aug 1.

21. Loibl S, Schwedler K, Von Minckwitz G, Strohmeier R, Mehta KM, Kaufmann $\mathrm{M}$. Venlafaxine is superior to clonidine as treatment of hot flashes in breast cancer patients - a double-blind, randomized study. Annals of oncology. 2007;18(4):689-693.

22. Towlerton G, Filshie J, O’Brien M, Duncan A. Acupuncture in the control of vasomotor symptoms caused by tamoxifen. Palliat Med. 1999;13(5):445.

23. Hjermstad MJ, Fossa SD, Bjordal K, Kaasa S. A test/retest study of the European Organization for Research and Treatment of Cancer core qualityof-life questionnaire. L Clin Oncol. 1995;13(5):1249-1254.

24. Stahl SM. Nuevos antidepresivos y estabilizadores del estado de ánimo. Psicofarmacología esencial. $2^{\mathrm{a} e d . ~ 2002 ; ~ p .267-326 . ~}$

25. Davis R, Whittington R, Bryson HM. Nefazodone, a review of its pharmacology and clinical efficacy in the management of major depression. Drugs. 1997;53(4):608-636.

26. Owens MJ, Morgan WN, Plott SJ, Nemeroff CB. Neurotransmitter receptor and transporter binding profile of antidepressants and their metabolites. $J$ Pharmacol Exp Ther. 1997;283(3):1305-1322.

27. Stahl SM. Basic psychopharmacology of antidepressants, part 1: antidepressants have seven distinct mechanisms of action. J Clin Psychiatry 1998;59 Suppl 4:5-14.

28. Krege S, Goepel M, Sperling H, Michel MC. Affinity of trazodone for human penile alpha1- and alpha2- adrenoceptors. Drug Metab Dispos. 2000;85(7): 959-961.

29. Marek GJ, McDougle CJ, Price LH, Seiden LS. A comparison of Trazodone and fluoxetine: implications for a serotonergic mechanism of antidepressant action. Psychopharmacology. 1992;109(1-2):2-11.

30. Pansini F, Albertazzi P, Bonaccorsi G, Zanotti L, Porto S, Dossi L, et al Trazodone: a non-hormonal alternative for neurovegetative climateric symptoms. Clin Exp Obstet Gynecol. 1995;22(4):341-344.

31. Matarredona J, Pérez E, Bonete JM. Actividad del hidroclorhidrato de trazodona en los trastornos sexuales producidos por los antidepresivos ISRS Psiq Biol, 2005;12:8-13.

32. Patten SB. The comparative efficacy of trazodone and imipramine in the treatment of depression. CMAJ. 1992;146(7):1177-1182.

Correspondencia autor: Dr. Santiago Vilar González

Servicio de Radioterapia

Complejo Hospitalario Universitario de Albacete (CHUA)

Hnos. Falco, s/n - 02006 Albacete. Tef : 0034967597523

E-mail autor: santiagov06@gmail.com

Información artículo: Revisión - Cáncer de próstata

Trabajo recibido: enero 2009

Trabajo aceptado: febrero 2009 